\title{
La tecnología como factor de competitividad - recientes indicadores internacionales de desempeño
}

Claudia María Rodríguez-Argueta' Investigadora asociada Utec crodarg@gmail.com

Recibido: 05/05/2016 - Aprobado: 18/05/2016

\section{Resumen}

Ante la vertiginosidad de los avances tecnológicos, los procesos de investigación, desarrollo e innovación desempeñan un papel trascendental para las industrias salvadoreñas como factor de competitividad internacional; sin embargo, El Salvador es uno de los países a escala mundial que menos invierte en investigación y desarrollo (I\&D) en relación con la producción nacional. Otro indicador que visibiliza la brecha existente en el país en los temas de ciencia, tecnología e innovación es el rezago en el número de artículos en publicaciones científicas y técnicas.

Por otro lado, a escala global, se vislumbran tendencias económicas que infieren algunas ventanas de oportunidad, tales como la denominada cuarta revolución industrialdigital, en la cual las tecnologías de la información y comunicación jugarán un papel clave en la productividad y competitividad de las industrias y el comercio, así como en el desarrollo de nuevas actividades productivas de mayor intensidad tecnológica.

\section{Abstract}

Under rapid technological advances, the processes of research, development and innovation play a crucial role for Salvadoran industries as a factor of international competitiveness; however, El Salvador is one of the world economies with the least investment on research and development (R\&D) in relation to GDP. Another indicator that makes perceptible the country's gap on science, technology and innovation is the lag in the number of articles in scientific and technical publications.

On the other hand, globally, an economic trend and potential opportunity is the so-called fourth industrial-digital revolution; in which, the information and communication technologies (ICT) will play a key role in the productivity and competitiveness of many sectors, as well as in the development of new activities of greater technological intensity. In the Network Readiness Index 2015 of the World Economic Forum, El Salvador upgraded its world ranking by escalating from the 93th position to the 80th

1 Investigadora asociada Utec. 
En el Índice Global de Tecnología de la Información 2015, del Foro Económico Mundial, El Salvador mejoró su ranking mundial, pasando de la posición 93 a la 80; entre 143 países evaluados. Empero, se deberán ir mejorando las condiciones claves en este índice, tales como el acceso a internet en la población, para ir reduciendo la brecha digital a escala nacional, así como la necesidad de una mayor inversión en I\&D en los sectores empresarial y académico y, paralelamente, la implementación de nuevas políticas de desarrollo para ir fortaleciendo la economía digital y la sociedad del conocimiento, que conlleven hacia una transformación productiva en el país.

\section{Palabras clave}

Ciencia, tecnología, innovación, competitividad, investigación y desarrollo among 143 countries. However, the key terms in this index such as Internet access in the population, to reduce the digital gap should improve. As well as, the need for greater investment in R\&D in the business and academic sectors and, at the same time, the need of renovated public policies to strengthen the digital economy and the knowledge society that could lead to a productive transformation in El Salvador.

\section{Keywords}

Science, technology, innovation, competitiveness, research and development
En la actualidad, existen diversos factores de cambio que afectan y seguirán impactando de forma estructural las actividades productivas a nivel mundial, entre los principales destacan:

1. La fragmentación, la especialización y la relocalización de las cadenas globales de producción.

2. La reducción de los costos de transporte y de comunicación (incluyendo la asequibilidad de las tecnologías de la información y comunicación) que permiten una mayor y mejor interacción de las empresas en el comercio internacional.

3. Los nuevos modelos de negocios que surgen a raíz de los rápidos cambios tecnológicos.
4. Nuevos ámbitos económicos tales como la denominada Cuarta Revolución Industrial - Digital o Industria $4.0^{2}$

En este informe abordaremos los últimos tres puntos que refieren a la vertiginosidad de los cambios tecnológicos y como El Salvador podría aprovechar algunas ventanas de oportunidad ante estos factores de cambio. Para ello será importante evaluar diversos indicadores de competitividad relacionados a los temas de ciencia, tecnología e innovación, con el objetivo de identificar brechas en áreas específicas $y$, desde la perspectiva académica, delinear algunas recomendaciones generales y políticas de desarrollo.

2 Industria 4.0- Es un enfoque innovador para alcanzar nuevos resultados gracias a los avances en la tecnología. Se espera que esta fase de la manufactura impulse cambios fundamentales disruptivos, razón por la que se le llama la cuarta revolución industrial. Su motor es el internet, y está compuesto por varios elementos como big data analytics, almacenamiento en la nube, infraestructura de las comunicaciones, Realidad Aumentada, movilidad, robótica e Internet de las cosas (Internet of Things), entre otros. Como resultado se obtienen procesos de fabricación más flexibles, mayor control e información en tiempos reales y se facilita la personalización masiva de los productos y servicios, generando mayor valor agregado. 


\section{A. ¿Cuánto invertimos en investigación y desarrollo (I\&D) en el país?}

El rápido y cambiante progreso tecnológico conjunto con el dinamismo del comercio mundial implica que el nivel de competitividad de las industrias dependerá, en gran medida, de su habilidad de innovar y de su capacidad de captar y adoptar tecnología. El nuevo entorno competitivo pone fuerte énfasis en las actividades de investigación, desarrollo e innovación (I\&D+i), no solo como base del crecimiento económico para los países de ingresos altos; también, se vuelve imperativo para las economías en vías de desarrollo con el fin de incrementar los niveles de eficiencia y, posteriormente, realizar "el salto" hacia la creación de nuevas industrias de mayor intensidad tecnológica.

El Salvador pertenece al grupo de países denominados como "naciones con sistemas incipientes de I\&D", que incluye también a Nicaragua, Perú, Paraguay, Guatemala, Bolivia, Ecuador, Republica Dominicana y Honduras. Este grupo se caracteriza precisamente porque sus sistemas de I\&D se reduce, en forma limitada, a algunas instituciones gubernamentales e Institutos de Educación Superior (IES); la participación del sector empresarial es casi nula en este bloque de países (Altenburg, 2000). En este grupo, los indicadores de gasto en actividades de I\&D se encuentran valuados muy por debajo a los índices del promedio mundial.

Por ejemplo, el gasto en $1 \& \mathrm{D}^{3}$ es un indicador que mide la contribución de actividades de I\&D como porcentaje del Producto Interno Bruto (PIB). Para el periodo 2011-2012, el promedio de gasto en I\&D a nivel mundial fue de $2.18 \%$, y el de los países en desarrollo de América Latina y el Caribe fue de $0.76 \%$. El Salvador invierte muy por debajo del promedio mundial y regional en I\&D en general, $0.03 \%$ versus el $2.18 \%$ y $0.76 \%$ respectivamente.

Gráfico 1. Gasto en I\&D - Grupo de países seleccionados 2011-2012 (Porcentaje del PIB)

\begin{tabular}{|c|c|c|}
\hline \multirow[b]{2}{*}{ Mundo } & \multicolumn{2}{|c|}{ 2011-2012 } \\
\hline & & 2.18 \\
\hline Latinoamérica & [ & 0.76 \\
\hline Países de ingreso mediano bajo & & 0.58 \\
\hline Costa Rica & 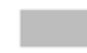 & 0.47 \\
\hline Panamá & 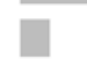 & 0.18 \\
\hline Guatemala & | & 0.05 \\
\hline El Salvador & | & 0.03 \\
\hline Honduras & & n.d \\
\hline Nicaragua & & n.d \\
\hline
\end{tabular}

Fuente: Sistema de Indicadores Utec con base a cifras del Banco Mundial. Nota: No existen datos disponibles para Nicaragua y Honduras.

3 Los gastos en investigación y desarrollo son gastos corrientes y de capital (público y privado) en trabajo creativo realizado sistemáticamente para incrementar los conocimientos, incluso los conocimientos sobre la humanidad, la cultura y la sociedad, y el uso de los conocimientos para nuevas aplicaciones. El área de investigación y desarrollo abarca la investigación básica, la investigación aplicada y el desarrollo experimental (Banco Mundial). 


\section{B. Artículos en publicaciones científicas y técnicas}

Otro indicador relevante para evaluar el factor de competitividad - ciencia, tecnología e innovación, es el número de artículos difundidos del país en publicaciones científicas y técnicas. Con base a la definición del Banco Mundial, los artículos en publicaciones científicas y técnicas se refieren a la serie de informes científicos y de ingeniería en los siguientes campos: física, biología, química, matemática, medicina clínica, investigación biomédica, ingeniería y tecnología, y ciencias de la tierra y el espacio. Estas publicaciones constituyen el instrumento imprescindible para impulsar la difusión social del conocimiento científico y tecnológico. La edición de revistas científicas en Iberoamérica es un medio para dar a conocer los resultados alcanzados por la investigación que se desarrolla en los países de la región. Por lo tanto, identificar, preservar, estimular y apoyar aquellas revistas que en cada país conforman el núcleo más selecto de su producción científica es un instrumento de fundamental importancia para fortalecer el proceso de creación de conocimientos, favorecer su difusión social y propiciar una cultura de integración regional (Albornoz, 2006).

Con base a cifras del Banco Mundial, El Salvador ocupa la posición cuarta de los seis países de la región centroamericana en el número de artículos de publicaciones científicas y técnicas. Para el 2013, el número de artículos reconocidos en publicaciones científicas y técnicas sumaron 35 informes en el país; cifra muy por debajo al no. de artículos que publica Costa Rica, la cual asciende a 277.

Gráfico 2. Artículos en publicaciones científicas y técnicas Grupo de países seleccionados 2013

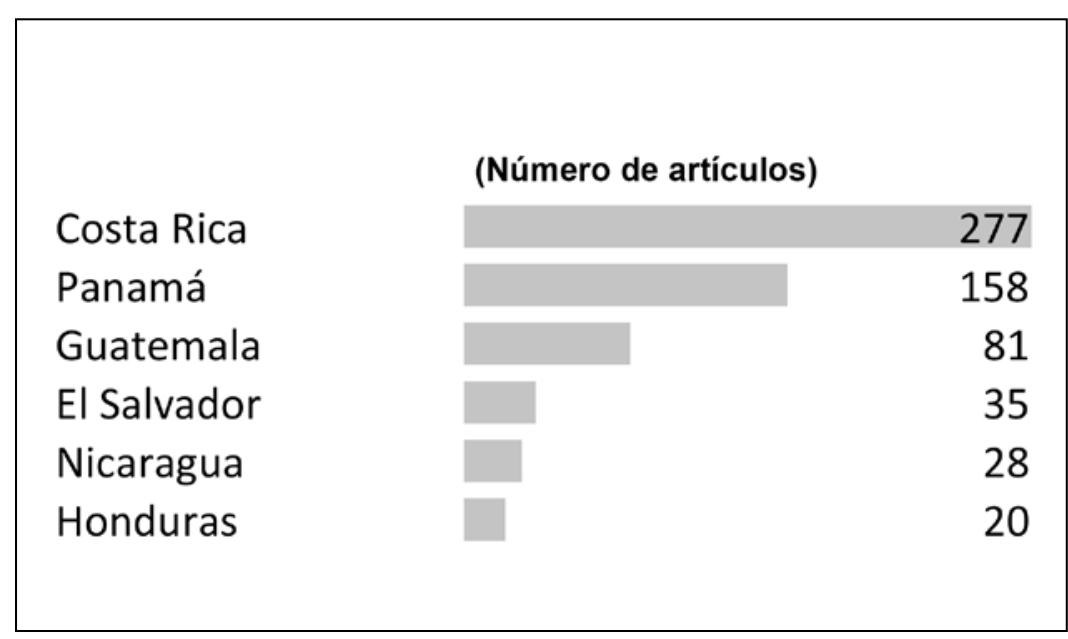

Fuente: Sistema de Indicadores Utec con base a cifras del Banco Mundial.

Según Albornoz, el fortalecimiento de las publicaciones científicas requiere también el estímulo y apoyo de las instituciones que rigen la política científica y tecnológica del país, así como de las entidades de docencia e investigación correspondientes. Es necesario apoyar a las publicaciones científicas por medio de un sistema concursable de financiamiento y premios a la calidad editorial y científica. También es imprescindible estimular la coordinación de esfuerzos entre los editores, las instituciones y la propia comunidad científica. 


\section{Las tecnologías de la información} y comunicación (TIC) como catalizadores del desarrollo

Según un estudio reciente elaborado por la empresa consultora en tecnología International Data Corporation (IDC, 2014), el mercado global TIC se encuentra en una fase de cambio denominada la "Tercera Plataforma". Esta nueva fase basada en cuatro pilares: Cloud Computing, Big Data, Mobility y Social Business, modificará para siempre el ecosistema empresarial. IDC espera que para el año 2020 las empresas inviertan más del $45 \%$ de su presupuesto de TI en la Tercera Plataforma. Las empresas cada día reconocen la importancia de invertir en soluciones tecnológicas que los acerquen a esta fase, como una estrategia que les permitirá obtener mayor competitividad en los negocios.

IDC infiere que la industria TIC ha evolucionado de una Primera y Segunda Plataforma, que se caracterizaba por una penetración TIC por habitante de menos $10 \%$ de la población. La primera plataforma tecnológica nace durante los años 60 , con los primeros sistemas informáticos utilizados por entidades gubernamentales estadounidenses para el procesamiento de información de manera centralizada: los mainframes. Más tarde, en la década de los 80 , con la aparición de la PC surge la segunda plataforma. Y en la actualidad, la nueva fase se caracteriza por una penetración TIC de mil millones de usuarios (más del $40 \%$ de la población mundial con acceso) y millones de aplicaciones, data y contenidos digitales desarrollándose día tras día.
Este fenómeno denominado por algunos economistas como una nueva revolución industrial-digital brinda una oportunidad para los países en desarrollo a vincularse a nichos catalizadores del crecimiento económico. Muchas naciones que han identificado las TIC como una industria clave para fomentar el crecimiento económico, y siendo el capital humano el factor critico de éxito para desarrollar ventajas competitivas en esta industria, están realizando programas y políticas públicas en que interactúan el sector empresarial, las Instituciones de Educación Superior (IES) y el gobierno para implementar las acciones necesarias para el desarrollo de los diferentes factores que componen el eco-sistema TIC.

El Salvador ha logrado progresar sustancialmente en algunos indicadores internacionales de competitividad relacionados a las TIC como lo es el Network Readinesss Index 2015 del Foro Económico Mundial (FEM); en donde el país avanzó de la posición 93 a la 80 entre 143 países evaluados. El FEM con el Informe Global de Tecnología de la Información (TIC) pretende explorar el impacto de dichas tecnologías en la productividad y el desarrollo de cada país. En este informe se incluye el Network Readiness Index (NRI por sus siglas en ingles) que, en los últimos quince años, ha medido el grado en el que las economías de todo el mundo aprovechan las TIC para mejorar su competitividad. Este índice basa su análisis en 10 pilares que le permiten establecer, con base a encuestas de percepción, el grado de preparación tecnológica con el que cuenta un país y, con base en ello, medir la relación existente entre el uso de TIC y su desempeño económico (Ramírez, 2013).

Gráfica 3. Ranking- Network Readiness Index, región centroamericana 2015

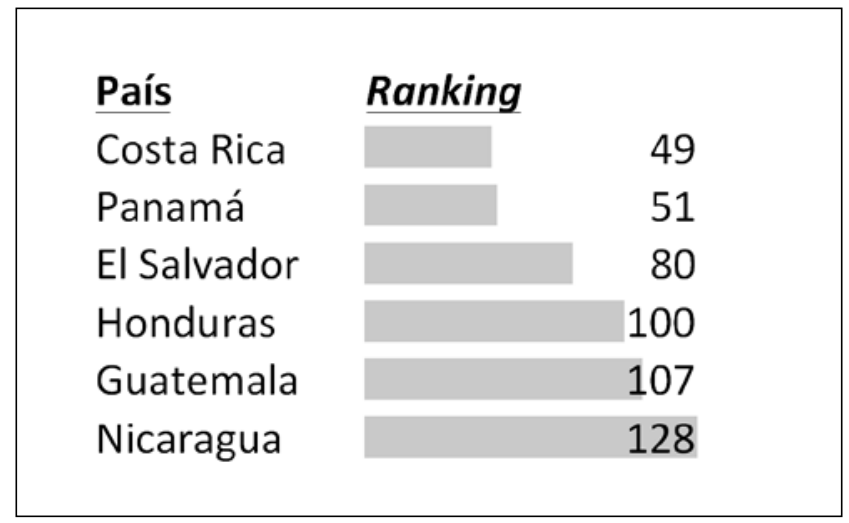

Fuente: Sistema de Indicadores Utec con base a cifras del Foro Económico Mundial. 
Sin embargo, el desempeño competitivo TIC, en relación con terceros países, es débil en El Salvador y existe mucho por realizar para aprovechar la denominada economía digital ${ }^{4}$. Por ejemplo, el acceso a internet es muy limitado en el país si la comparamos a las economías pares. A continuación se presenta un comparativo regional de los usuarios de internet con acceso a la red mundial; este indicador mide el número de usuarios por cada 100 personas por país. En el caso de El Salvador, existe una proporción de 29.70 usuarios por cada 100 personas; cifra muy por debajo del promedio mundial y regional (Ver Gráfica 4).

\section{Gráfica 4. Usuarios de internet - Comparativo regional - 2014 (por cada 100 personas)}

\begin{tabular}{|l|l|}
\hline & \\
América Latina y el Caribe & 50.15 \\
Costa Rica & 49.41 \\
Panamá & 44.92 \\
Mundo & 40.69 \\
El Salvador & 29.70 \\
Guatemala & 23.40 \\
Países de ingreso mediano bajo & 22.56 \\
Honduras & 19.08 \\
Nicaragua & 17.60 \\
\hline
\end{tabular}

Fuente: Sistema de Indicadores Utec con base a cifras del Banco Mundial.

Es importante señalar que el nivel de impacto económico de las TIC está en relación a su capacidad de generación de valor en otras actividades económicas y, para ello, es fundamental la transformación productiva de la misma. Reducir la brecha digital en El Salvador debería ser una apuesta-país y una estrategia nacional para su desarrollo económico-social.

\section{Hacia una transformación productiva en actividades de mayor intensidad tecnológica}

Otro indicador del factor de competitividad en ciencia, tecnología e innovación es la intensidad tecnológica de la producción industrial. En el nuevo entorno, los procesos de industrialización han cambiado respecto a décadas pasadas, dado que la tecnología y el conocimiento juegan un rol vital en la productividad total de los factores de producción. La mayor dependencia de productos manufactureros con mayor valor tecnológico y ligados con actividades de servicios más sofisticados (ej. diseño, logística, tecnologías de la información, $1 \& D+i)$ torna al país menos vulnerable ante la competencia internacional.

Actualmente, la industrialización es vista como la especialización de procesos y servicios industriales $y$ tecnológicos más que la fabricación de un producto per se en regiones específicas, en donde la fragmentación e internacionalización de los procesos productivos convierte a la exportación de productos manufacturados en uno de los mejores medios para beneficiarse de la globalización (ONUDI, 2013).

4 Según CEPAL (2013), la economía digital está constituida por la infraestructura de telecomunicaciones, las industrias TIC (software, hardware y servicios TIC) y la red de actividades económicas y sociales facilitadas por internet, la computación en la nube (cloud computing) y las redes móviles, las sociales y de sensores remotos. 
Con base a la definición de la Organización de las Naciones Unidades para el Desarrollo Industrial (ONUDI), Ios productos de media y alta intensidad tecnológica requieren de procesos de alto valor agregado dentro de la cadena productiva industrial. Por ejemplo, los productos calificados como de media intensidad tecnológica (MT) requieren de procesos de aprendizaje prolongados, capacidades técnicas y organizaciones, así como la habilidad de manejar procesos intensivos en escala; en cambio, los bienes de alta intensidad tecnológica (AT) requieren de competencias muy avanzadas en innovaciones tecnológicas. Las exportaciones de productos de alta tecnología son productos altamente intensivos en investigación y desarrollo, como son los productos de las industrias aeroespacial, informática, farmacéutica, de instrumentos científicos y de maquinaria eléctrica.

En la siguiente gráfica 5 , se muestra como El Salvador se encuentra por debajo de la media regional y mundial en el porcentaje de las exportaciones de productos manufacturados de alta tecnología, equivalente a $4.76 \%$ para el año 2014 según indicadores del Banco Mundial.

\section{Gráfica 5. Exportaciones de productos de alta tecnología, grupo de países seleccionados 2014 (\% de las exportaciones de productos manufacturados)}

\begin{tabular}{|l|r|}
\hline & $\mathbf{2 0 1 4}$ \\
Costa Rica & 43.42 \\
Mundo & 18.44 \\
Países de ingreso mediano bajo & 11.55 \\
América Latina y el Caribe & 10.90 \\
Guatemala & 4.96 \\
El Salvador & 4.76 \\
Honduras & 2.42 \\
Nicaragua & 0.39 \\
Panamá & 0.20 \\
\hline
\end{tabular}

Fuente: Sistema de Indicadores Utec con base a cifras del Banco Mundial.

Es importante que en la actividad productiva en El Salvador se dinamicen la producción de rubros de mayor intensidad tecnológica. Según ONUDI, las actividades de alta intensidad tecnológica son menos vulnerables a la entrada de competidores que las de baja intensidad tecnológica, cuyos requerimientos de escala y capacidades son también menores. Sectores basados en recursos naturales y de baja tecnología constituyen los puntos de entrada de países en desarrollo. Es por esto que en dichos sectores las rentas industriales son más bajas y están más expuestas a la competitividad internacional. Por último, los sectores con alto componente tecnológico ofrecen mayores perspectivas para el aprendizaje y la innovación. Por lo tanto, manteniendo iguales a todos los otros factores, la tecnología posibilita un crecimiento más rápido en las capacidades de mayor calidad.

\section{Algunas recomendaciones:}

Algunas de las recomendaciones iniciales para impulsar las áreas de ciencia, tecnología e innovación como factor de competitividad es:

1. Una participación más activa de las IES privadas en la Política Nacional de Ciencia, Tecnología e Innovación.

2. Otro factor relevante es una mayor y mejor vinculación de las IES con las empresas para los proyectos de investigación y desarrollo. Es trascendental estimular el acercamiento entre ambas partes con el objetivo de establecer puntos de contacto para identificar necesidades y brindar soluciones tecnológicas a 
los sectores productivos a través de una mayor vinculación de las necesidades de I\&D de las empresas con las actividades y capacidad instalada de las IES en las áreas de ciencia y tecnología aplicada.

3. Por otro lado, es necesario apoyar las publicaciones científicas por medio de un sistema concursable de financiamiento y premios a la calidad editorial y científica y, a la vez, estimular la coordinación de esfuerzos entre los editores, las instituciones y la propia comunidad científica.

\section{Referencias}

Altenburg, T. (2000), "Linkages and Spillovers between Transnational Corporations and Small and MediumSized Enterprises in Developing Countries, Opportunities and Policies". Recuperado de: https:// www.researchgate.net/publication/237493279_Linkages_ and_Spillovers_between_Transnational_Corporations_ and_Small_and_Medium-Sized_Enterprises_in Developing_Countries_-_Opportunities_and_Policies

Gaillard, J. (2010), Measuring R\&D in Developing Countries: Main Characteristics and Implications for the Frascati Manual. Recuperado de: http://horizon. documentation.ird.fr/exl-doc/pleins_textes/ divers11-04/010051097.pdf
UNESCO (2010). Measuring R\&D: Challenges Faced by Developing Countries. Recuperado de: http://www. uis.unesco.org/Library/Documents/tech\%205-eng.pdf

Albornoz, M (2006). Estrategias para la promoción de las publicaciones científicas argentinas. Recuperado de: http://www.scielo.org.ar/scielo.php?script=sci_artte xt\&pid=S0325-29572006000200012

Dutta, S., Geiger, T., \& Lanvin, B. (Eds.). (2015). The global information technology report 2015. ICTs for inclusive growth. Foro Economico Mundial. Recuperado de http://www3.weforum.org/docs/WEF_Global_IT_ Report_2015.pdf

Organización de las Naciones Unidas para el Desarrollo Industrial. (ONUDI, 2013). The industrial competitiveness of nations: Looking back, forging ahead. Competitive industrial performance report 2012/2013. Recuperado de: https://www.unido.org/ fileadmin/user_media/Services/PSD/Competitive Industrial_Performance_Report_UNIDO_2012_2013. PDF

Organización de las Naciones Unidas para el Desarrollo Industrial. (UNIDO, 2015). The role of technology and innovation in inclusive and sustainable industrial development. Industrial development report 2016. Recuperado de: https://www.unido.org/fileadmin/ user_media_upgrade/Resources/Publications/ EBOOK_IDR2016_FULLREPORT.pdf 